EPJ Web of Conferences 55, 02002 (2013)

DOI: $10.1051 /$ epjconf/20135502002

(C) Owned by the authors, published by EDP Sciences, 2013

\title{
Monte Carlo methods
}

\author{
Rémi Bardenet \\ ${ }^{1}$ Department of Statistics, Oxford University
}

\begin{abstract}
Bayesian inference often requires integrating some function with respect to a posterior distribution. Monte Carlo methods are sampling algorithms that allow to compute these integrals numerically when they are not analytically tractable. We review here the basic principles and the most common Monte Carlo algorithms, among which rejection sampling, importance sampling and Monte Carlo Markov chain (MCMC) methods. We give intuition on the theoretical justification of the algorithms as well as practical advice, trying to relate both. We discuss the application of Monte Carlo in experimental physics, and point to landmarks in the literature for the curious reader.
\end{abstract}

\section{Introduction}

Bayesian statistics (see B. Clément's and D. Sivia's contributions in this volume) quantify the degree of belief one has on quantities or hypotheses of interest, given the data collected. More precisely, let us assume that a model of an experiment is available under the form of a likelihood $p($ data $\mid x)$, and that one has chosen a prior $p(x)$ on the parameters $x \in \mathbb{X} \subset \mathbb{R}^{d}$ of the experiment. Then all knowledge on $x$ is encoded by the posterior distribution

$$
\pi(x)=p(x \mid \text { data })=\frac{p(\text { data } \mid x) p(x)}{\int p(\text { data } \mid x) p(x) d x} .
$$

To summarize the inference, one might, for example, want to compute the mean posterior estimate

$$
x_{\mathrm{MEP}}=\int x \pi(x) d x
$$

and report a credible interval $C$ such that $x_{\mathrm{MEP}} \in C$ and

$$
\int_{C} \pi(x) d x \geq 95 \% .
$$

Both of these tasks require to be able to compute integrals with respect to $\pi$. While in some cases these integrals might be analytically tractable, they are usually not in experimental physics, since the likelihood $p($ data $\mid x)$ often takes a complex form, which $\pi$ inherits, as we shall now see in an example inspired by the Pierre Auger experiment.

This is an Open Access article distributed under the terms of the Creative Commons Attribution License 2.0, which permits unrestricted use, distribution, and reproduction in any medium, provided the original work is properly cited. 


\subsection{A model inspired by Auger}

The Pierre Auger observatory ${ }^{1}$ is a large-scale particle physics experiment dedicated to the observation of atmospheric showers triggered by cosmic rays. These showers are wide cascades of elementary particles raining on the surface of Earth, resulting from charged nuclei hitting our atmosphere with the highest energies ever seen.

The surface detector of the Pierre Auger experiment (henceforth Auger) consists of water-filled tanks and their associated electronics - arranged on a triangular grid, the distance between two tanks being 1.5 kilometers, with the grid covering a total area of 3000 square kilometers. We model here the tankwise signal produced by one kind of particle in the shower: muons.

When a muon crosses a water tank, it generates Cherenkov photons and photons coming from other processes (e.g., delta rays) along its track at a rate depending on its energy. Some of these photons are captured by photomultipliers. The resulting photoelectrons (PEs) then generate analog signals that are discretized by an analog-to-digital converter.

In this section, we model the integer photoelectron (PE) count vector $\mathbf{n}=\left(n_{1}, \ldots, n_{M}\right) \in \mathbb{N}^{M}$ in the $M$ bins of the signal, which means that we omit the model of the electronics. Formally, $n_{i}$ is the number of PEs in the $i$-th bin

$$
\left[t_{i-1}, t_{i}\right)=\left[t_{0}+(i-1) t_{\Delta}, t_{0}+i t_{\Delta}\right),
$$

where $t_{0}$ is the absolute starting time of the signal, and $t_{\Delta}=25 \mathrm{~ns}$ is the signal resolution (size of one bin). The goal is to parametrize the likelihood $p(\mathbf{n} \mid t, A)$, where $t$ is the arrival time of the muon and $A$ is the integrated signal amplitude.

Given the arrival time $t$ of a muon and the associated total number of PEs $A$, the PE count in the $i$ th bin is a Poisson variable with parameter

$$
\bar{n}_{i}(t, A)=A \int_{t_{i-1}}^{t_{i}} r(s-t) d s .
$$

where the ideal unit response $r(\cdot)$ is given in Figure 1(a), the analytical expression being omitted for the sake of simplicity. Let the number $N_{\mu}$ of muons crossing the tank be known. Adding the contributions of $N_{\mu}$ muons with signal amplitudes $\mathbf{A}=\left(A_{1}, \ldots, A_{N_{\mu}}\right)$ and arrival times $\mathbf{t}=\left(t_{1}, \ldots, t_{N_{\mu}}\right)$, the binwise signal expectation is

$$
\bar{n}_{i}(\mathbf{t}, \mathbf{A})=\sum_{j=1}^{N_{\mu}} \bar{n}_{i}\left(t_{j}, A_{j}\right) .
$$

Let now $x=\left(t_{1}, A_{1}, \ldots, t_{N_{\mu}}, A_{N_{\mu}}\right)$. Our likelihood is finally

$$
p(\mathbf{n} \mid x)=\prod_{i=1}^{M} \operatorname{Poi}_{\bar{n}_{i}(\mathbf{t}, \mathbf{A})}\left(n_{i}\right) .
$$

A summary of the generating model is depicted in Figure 1(b).

This model is only a sketch of what is needed to achieve inference in Auger: in practice, more nuisance variables have to be added, to describe, e.g., the noise in the detector electronics. Still, even with simple flat priors on $x$, integrals with respect to the resulting posterior

$$
\pi(x) \propto p(\mathbf{n} \mid x) p(x)
$$

cannot be analytically computed.

\footnotetext{
${ }^{1}$ www.auger.org
} 


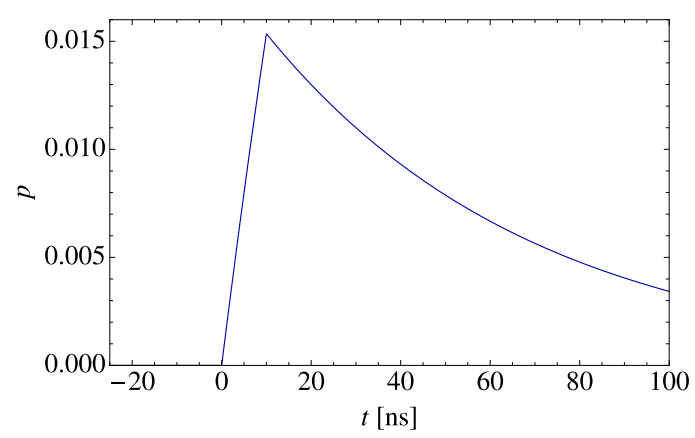

(a) The muonic time response model $r(t)$.

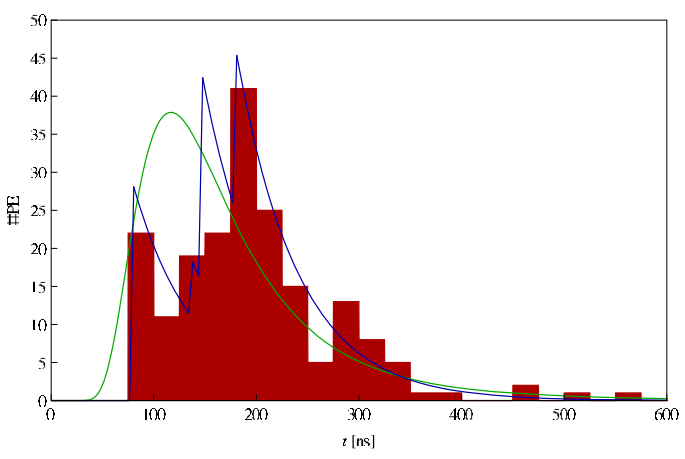

(b) An example signal

Figure 1. The generative model of the muonic signal. (a) Ideal unit response function $r(\cdot)$. (b) The green curve is the time-of-arrival distribution $p\left(t_{\mu}\right)$ used to generate this example with $N_{\mu}=4$. The amplitude distribution is not shown, but derives from the geometry of the tank. The blue curve is the ideal response $\sum_{j=1}^{4} A_{j} r\left(t-t_{j}\right)$, and the red histogram is the signal (PE count vector) $\mathbf{n}$.

\subsection{The Monte Carlo principle}

Since integrals like (2) cannot be analytically derived, we have to rely on numerical approximation techniques. The cost of classical non-probabilistic numerical integration based on regular grids grows exponentially with the dimension $d$. The rationale behind Monte Carlo (MC) methods is to replace grids by stochastic samples. Let first

$$
\widehat{I}_{N}=\frac{1}{N} \sum_{i=1}^{N} h\left(X_{i}\right) .
$$

If $X_{1}, \ldots, X_{N} \sim \pi$ are independent and identically distributed (i.i.d.), then

$$
\widehat{I}_{N} \approx I=\int h(x) \pi(x) d x
$$

Note that $\widehat{I}_{n}$ in (6) is a random variable. Its expectancy is precisely $I$ (we say $\widehat{I}_{N}$ is an unbiased estimator or $I$ ) and its variance is $V / N$, where $V$ is the variance of $h(X)$ when $X \sim \pi$. This justifies the saying that Monte Carlo error decreases as $\sqrt{N}$.

Interestingly, one can see the MC principle as the randomization of a grid method: points are not regularly spread across the space anymore, but sampled according to $\pi$. This is intuitively efficient, since regions of the space should be examined all the more finely that they contribute to the integral $I$. In other words, putting a fine grid where $\pi$ is large and a scarce one where $\pi$ is small will yield to an estimator $\widehat{I}_{N}$ with small variance.

What makes a good MC method is thus its ability to sample from $\pi$. In this tutorial, we describe various ways of sampling according to $\pi$, exactly or approximately. Note that the sampling methods we describe are generic and can find other, non-Bayesian applications in experimental physics: simulators like CORSIKA [15] implement sampling from complex, hierarchized distributions with MC methods.

Finally, a generic MC method should require only that $\pi$ is known up to a normalization constant, since in most applications, the denominator of (1) is intractable. 
The rest of this tutorial is organized as follows. In Section 2 we review basic non-MC sampling methods and MC methods that are based on i.i.d. sampling. The latter are useful in small dimensions (say smaller than 10) and often require that $\pi$ can be somehow approximated. Section 3 describes Markov chain Monte Carlo methods (MCMC). MCMC methods generate a dependent sample which asymptotically resembles a sample from $\pi$. Section 4 presents advanced MCMC tools that learn or exploit the structure of $\pi$ for better sampling.

\section{First sampling methods}

\subsection{The inverse cdf method}

If the cumulative distribution function $F$ of $\pi$ is known and can be inverted, then it is enough to know how to sample $U$ from a uniform distribution ${ }^{2}$ on $[0,1]$. Indeed one can show that $F^{-1}(U)$ is then distributed according to $\pi$. This can be applied to generate exponential variables, for example. However, this method is not applicable beyond simple distributions, since we usually cannot even compute $F$, as it requires integrating with respect to $\pi$.

\subsection{The transformation method}

It is sometimes possible to obtain samples from $X \sim \pi$ by applying a transformation to variables $Y$ that are easier to sample. For example, building on the exponential generator of Section 2.1, we can add two independent exponential variables with parameter 1 to obtain a Gamma variable with parameters $(2,1)$, as is easily proven by a convolution. Again, this method is limited in its applications to simple distributions.

\subsection{Rejection sampling}

Rejection sampling is one of the simplest MC algorithms. It requires the knowledge of a distribution $q$ on $\mathbb{X}$ which is easy to sample from, such as a Gaussian or a Gamma distribution, and a constant $M>0$ such that

$$
\pi \leq M q .
$$

It works by repeatedly sampling according to a proposal distribution $q$ and accepting or rejecting each sample with a certain probability that guarantees that the final accepted samples are distributed according to $\pi$. The algorithm is presented in Figure 2.

Note that the tighter the bound in the right-hand side of (7), the less samples are rejected. Thus, a good knowledge of $q$ and $M$ is necessary for rejection sampling to be efficient. When such a bound is not known, one can resort to importance sampling.

\subsection{Importance sampling}

Importance sampling takes as input a proposal $q$ but does not require (7), only that $q$ puts mass wherever $\pi$ does. Furthermore, it only requires that $\pi$ is known up to a normalization constant. For clarity, we denote by $\pi_{0}$ the available unnormalized version of $\pi$, and will write $\pi$ only for the normalized target distribution.

\footnotetext{
${ }^{2}$ We shall assume here that a uniform generator is available, the good old rand() function. However, implementing such a generator is not trivial [18, Section 2.6 and references therein].
} 


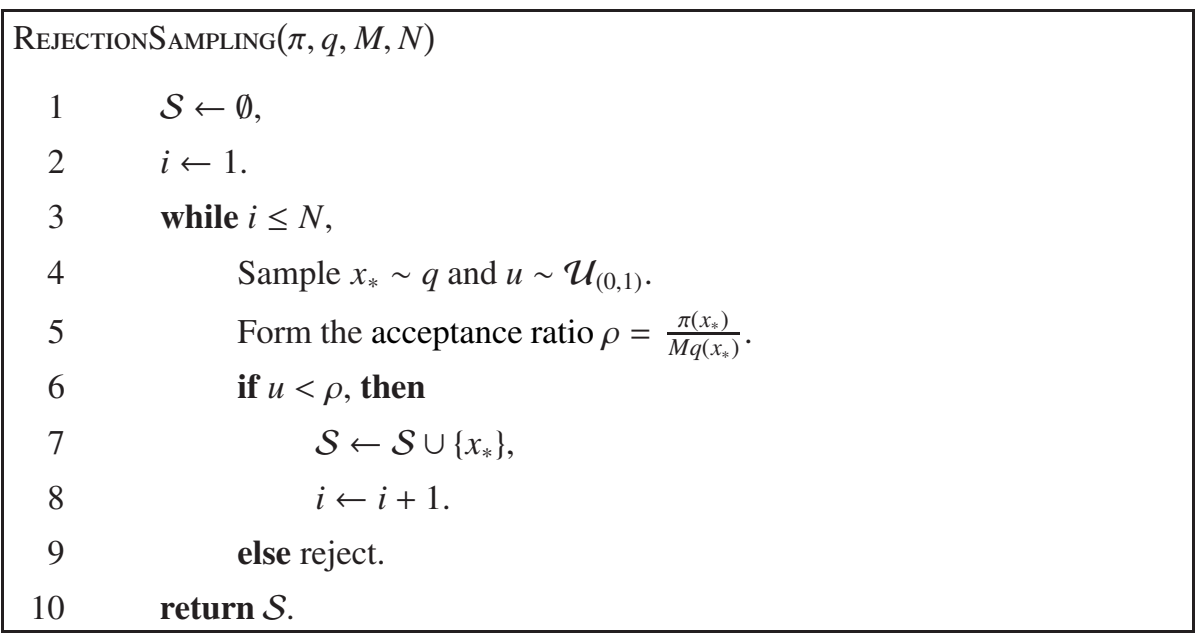

Figure 2. The pseudocode of the rejection sampling algorithm. The number of iterations to reach $N$ samples from $\pi$ is unknown beforehand and depends on the tightness of the bound in (7).

Unlike other algorithms in this chapter, each of which yields a sample $\mathcal{S}$, importance sampling return a weighted sample or, equivalently, an approximation of the target as weighted sum of point masses. Importance sampling is based on the law of large numbers [13, Section 7.5] with a reweighting trick. Precisely, importance sampling approximates the integral $I$ of (6) with the estimator

$$
\widetilde{I}_{N}=\frac{1}{Z} \sum_{i=1}^{N} w_{i} h\left(x_{i}\right)
$$

where

$$
w_{i}=\frac{\pi_{0}\left(x_{i}\right)}{q\left(x_{i}\right)}, \quad \text { and } \quad Z=\sum_{j=1}^{N} w_{j}
$$

In general, the estimator $\widetilde{I}_{N}$ is not unbiased, but only asymptotically unbiased. Indeed, applying the law of large numbers to both the numerator and the denominator, we obtain

$$
\widetilde{I}_{N}=\frac{\sum_{i=1}^{N} w_{i} h\left(x_{i}\right)}{\sum_{j=1}^{N} w_{j}} \rightarrow \frac{\int h(x) \pi_{0}(x) d x}{\int \pi_{0}(x) d x}=\int h(x) \pi(x) d x, \quad x_{i} \sim q \text { i.i.d. }
$$

where the convergence is almost sure ${ }^{3}$. Note, however, that if $\pi_{0}=\pi$ is normalized, one can replace $Z$ by $N$ in (8) and obtain an unbiased estimator $\widetilde{I}_{N}$. The pseudocode of the importance sampling algorithm is given in Figure 3.

To understand the rôle of the proposal $q$, it is useful to derive the asymptotic behaviour of the variance of the estimator:

$$
\operatorname{Var}\left(\widetilde{I}_{N}\right)=\frac{\sigma_{\lim }^{2}}{N}+o\left(\frac{1}{N}\right)
$$

\footnotetext{
${ }^{3}$ There are several modes of convergence for sequences of random variables, cf. [13, Section 7.2] for a summary.
} 
with

$$
\sigma_{\lim }^{2}=\int[h(x)-I]^{2} \frac{\pi(x)}{q(x)} \pi(x) d x .
$$

Thus, in order to keep the variance of $\widetilde{I}_{N}$ - in physical terms the square of the statistical error low, $q$ has to be chosen close to $\pi$, and with heavier tails than $\pi$. The last requirement means that we must ensure that

$$
\sup _{x \in \mathbb{X}} \frac{\pi(x)}{q(x)}<\infty
$$

\begin{tabular}{|c|c|}
\hline \multicolumn{2}{|c|}{$\operatorname{IMPORTANCESAMPLING}\left(\pi_{0}, q, N\right)$} \\
\hline 1 & Sample independent $x_{i} \sim q, i=1, \ldots, N$ \\
\hline 2 & Form the weights $w_{i}=\frac{\pi_{0}\left(x_{i}\right)}{q\left(x_{i}\right)}$, \\
\hline 3 & Compute the normalization constant $Z=\sum_{i=1}^{N} w_{i}$, \\
\hline 4 & $\pi$ is approximated by $\frac{1}{Z} \sum_{i=1}^{N} w_{i} \delta_{x_{i}}$. \\
\hline
\end{tabular}

Figure 3. The pseudocode of the importance sampling algorithm only requires that $\pi$ is known up to a normalization constant. Unlike rejection sampling, no sample is wasted.

\subsubsection{On the choice of the proposal for importance sampling}

In practice, either a reasonable choice for $q$ is available, or not. The first case occurs when, e.g., $\pi$ is almost unimodal and concentrates its mass on a small region of $\mathbb{X}$. A Gaussian centered at this small region with a reasonable variance then yields a good choice for $q$. Easy-to-sample, heavytailed distributions like Student's distribution, are also handy. We have often seen the case in particle physics where $\pi$ is a posterior that puts all its mass on a small region of $\mathbb{R}^{d}$. In that case, remember that importance sampling with the right $q$ yields better accuracy than grid-based methods or uniform sampling.

If the choice of $q$ is not obvious, we recommend the use of an adaptive strategy, such as population Monte Carlo. A description of population MC and an application to model selection in cosmology can be found in [24]. Basically, first make a wild guess $q^{(0)}$ for $q$, say a Gaussian with a large variance. Apply importance sampling a first time to obtain an estimate of $\pi$ and fit a Gaussian $q^{(1)}$ to this estimate of $\pi$. Now re-apply importance sampling with $q^{(1)}$ as a proposal, and re-fit a new Gaussian $q^{(2)}$ to $\pi$, etc. After $T$ iterations, $q^{(T)}$ should be a good proposal distribution for importance sampling. Of course, you can apply this procedure with other candidate proposals than Gaussians, you should indeed choose a family of distributions among which you think you may find a good approximation of $\pi$. If you have reasons to believe that $\pi$ is bimodal, for example, you should probably fit a mixture of two distributions as in [24] rather than a Gaussian, which is unimodal. Usually, with the right choice of family of distributions, a few iterations are enough to get a reasonable $q$, and you can stop when $q^{(t)}$ does not change a lot with $t$.

A similar method for adaptively tuning the proposal in importance sampling has been quite popular in physics: nested sampling, on which we recommend [22]. Be careful, however, a common mistake is to forget the assumption that the final $q$ has to put mass wherever $\pi$ may. 


\subsubsection{Convergence diagnostics and confidence intervals}

There are a variety of criteria to assess the good behaviour of an importance sampling estimator. An important thing to check is the empirical distribution of the weights $w_{i}$. If only a few of the weights are nonzero, the estimator $\widetilde{I}_{N}$ is based on too few points and thus has a large variance. It is thus desirable to obtain a weight distribution with a high number of large and comparable weights, which, again, is achieved by finding a good proposal $q$.

More formal quality measures also exist, such as the so-called effective sample-size $\mathrm{ESS}_{N}$ :

$$
\operatorname{ESS}_{N}=\left(\sum_{i=1}^{N}\left(\frac{w_{i}}{\sum_{j=1}^{N} w_{j}}\right)^{2}\right)^{-1}
$$

$\mathrm{ESS}_{N}$ ranges from 1 (when only one weight is nonzero) to $N$ (when all weights are equal). Roughly, $\mathrm{ESS}_{N}$ is telling how many of the samples $x_{1}, \ldots, x_{N}$ are really independent in the following sense: the accuracy of $\widetilde{I}_{N}$ is equivalent to the accuracy obtained with $\operatorname{ESS}_{N}$ samples that would be drawn directly from the real $\pi$.

Finally, it is possible to derive asymptotic confidence intervals for $\widetilde{I}_{N}-I$, since it can be first shown that

$$
\sqrt{N}\left(\widetilde{I}_{N}-I\right) \rightarrow \mathcal{N}\left(0, \sigma_{\lim }^{2}\right),
$$

where the convergence is in distribution, and second

$$
N \sum_{i=1}^{N}\left(\frac{w_{i}}{\sum_{j=1}^{N} w_{j}}\right)^{2}\left(h\left(X_{i}\right)-\sum_{k=1}^{N} \frac{w_{k}}{\sum_{j=1}^{N} w_{j}} h\left(X_{k}\right)\right)^{2} \rightarrow \sigma_{\lim }^{2}
$$

almost surely.

\subsection{Going to higher dimensions}

We now consider an insightful example on how rejection and importance sampling scale when the dimension $d$ of the ambient space grows. Consider a simple unit Gaussian target $\pi=\mathcal{N}\left(0, I_{d}\right)$, where $I_{d}$ is the $d \times d$ identity matrix. Say we are fortunate enough to know that $\pi$ is an isotropic Gaussian, but ignore its variance. A relevant choice of proposal would then be an isotropic Gaussian $q(x)=\mathcal{N}\left(0, \sigma^{2} I_{d}\right)$.

If applying rejection sampling, one must know beforehand that $\pi$ has variance upper bounded by some constant $\sigma^{*}$, and then choose $\sigma \geq \sigma^{*}$, in order to satisfy (7). This is already a very strong assumption, but there is worse: the fraction of accepted samples goes as $\sigma^{-d}$. This means that if $\sigma$ is not exactly 1, one should expect an exponentially small number of accepted samples with growing $d$. A similar curse of dimensionality happens with importance sampling: the variance of the weights is either infinite if $\sigma \leq \frac{1}{\sqrt{2}}$, or it goes as $\sigma^{d}$.

\subsection{Conclusion on rejection and importance sampling}

Rejection sampling is very easy to implement and can work very well in settings where the relevant information on the target is known, as is sometimes the case in simulators like CORSIKA [15]. Importance sampling is more generic and can deal with unnormalized targets, as is often the case in Bayesian data analysis. The efficiency of both methods depends on the design of the proposal distribution $q$, and both do not scale to high dimensions (say larger than 10). 


\section{MCMC basics}

Rejection and importance sampling are Monte Carlo methods based on an i.i.d. sample from a proposal distribution $q$. To tackle large dimensions, other methods have been devised that are based on a non-independent sampling: Markov chain Monte Carlo methods (MCMC). The prototype of MCMC methods is the Metropolis-Hastings algorithm, of which almost all MCMC algorithms are variants. Note that although we concentrate here on applications to inference, MCMC is also used in simulators like CORSIKA, see [8] for an example.

\subsection{The Metropolis-Hastings algorithm}

We first describe Metropolis' algorithm in Figure 4. It builds a random walk $\left(X_{i}\right)$ that explores $\mathbb{X}$, and eventually approximates independent samples from $\pi$.

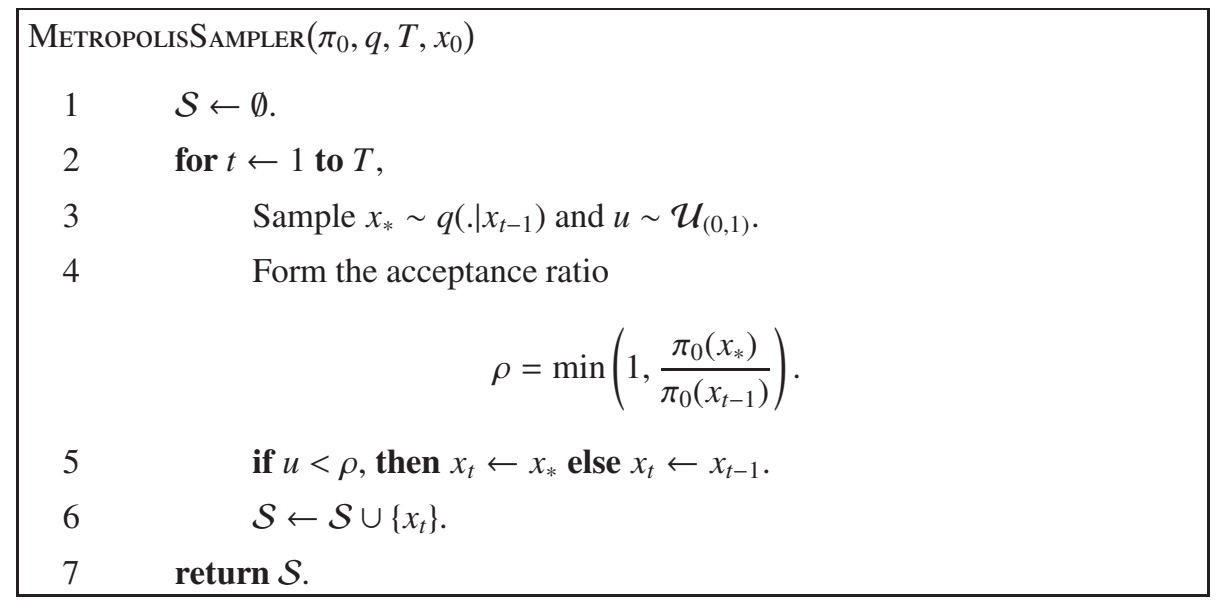

Figure 4. The pseudocode of the Metropolis algorithm.

Metropolis' algorithm is a for loop. At each iteration $t$, a candidate point $x^{*}$ is proposed in the neighborhood of the current position $x_{t-1}$, according to a proposal $q\left(\cdot \mid x_{t-1}\right)$. In Metropolis' algorithm, this proposal is assumed to be symmetric, i.e., $q(x \mid y)=q(y \mid x)$. In practice, a Gaussian with fixed covariance matrix $\Sigma$ is often used: $q(y \mid x)=\mathcal{N}(y \mid x, \Sigma)$. After the candidate point has been generated, it is accepted as the next position $x_{t}$ only with a certain probability $\rho$, which is 1 if $\pi\left(x^{*}\right)$ is larger than $\pi\left(x_{t-1}\right)$, and smaller than 1 (but often not zero!) if not. This precise definition of $\rho$ relates the random walk $\left(X_{i}\right)$ to $\pi$ and makes the algorithm different from an optimization algorithm: it does not always try to move for a point with larger $\pi$. Furthermore, the theory of Markov chains ${ }^{4}$ guarantees that such an acceptance rule implies that $\pi$ is the limiting distribution of the chain $\left(X_{i}\right)$, in a sense that shall become clear soon.

Now we are ready to present the Metropolis-Hastings $(\mathrm{MH})$ algorithm. It is simply Metropolis' algorithm, but with general, possibly nonsymmetric proposals. The pseudocode of $\mathrm{MH}$ is given in Figure 5. Note the new definition of the acceptance probability $\rho$, which ensures the final convergence to $\pi$. Intuitively, this acceptance rule cancels the influence of $q$ on the limiting distribution: the probability of accepting a move that $q$ is likely to draw often is reduced.

\footnotetext{
${ }^{4} \mathrm{~A}$ Markov chain is a sequence of random variables $\left(X_{i}\right)$ such that $X_{i+1}$ depends on the past only through $X_{i}$. More formally: $X_{i+1}\left|X_{1}, \ldots, X_{i} \sim X_{i+1}\right| X_{i}$.
} 


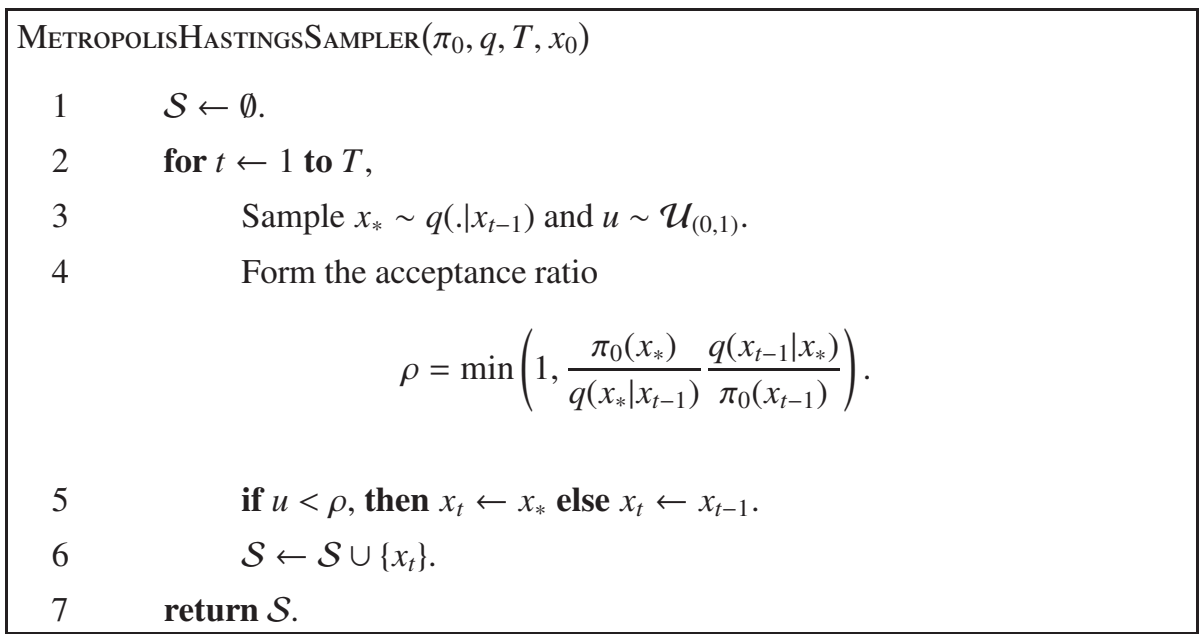

Figure 5. The pseudocode of the Metropolis-Hastings algorithm.

We refer the reader interested by a gentle introduction to theoretical results on $\mathrm{MH}$ to [18, Chapter 7]. We will limit ourselves to one simple but useful result, since it covers the common Metropolis algorithm with Gaussian proposals: assume $q(\cdot \mid x)$ puts mass over all $\mathbb{X}$ and that there exists $\delta>0$ and $\varepsilon>0$ such that

$$
\|x-y\|<\delta \Rightarrow q(y \mid x)>\varepsilon,
$$

then for any integrable function $h, \mathrm{MH}$ gives an asymptotically unbiased estimate of the integral I defined in (6):

$$
\lim _{N \rightarrow \infty} \widehat{I}_{N} \rightarrow I .
$$

This is an example of formal result that states that $\left(X_{i}\right)$ behaves like independent draws from $\pi$ for large $i$.

\subsection{Assessing convergence}

MH outputs a sample from a Markov chain that asymptotically approximates independent draws from $\pi$. From a practitioner's point of view, two questions arise, which we address successively in the rest of this section. First, since we are waiting for the chain $\left(X_{i}\right)$ to converge to $\pi$ whatever starting value $x_{0}$ we input, it is important to know from which iteration on the chain is independent from $x_{0}$. Second, even if the iteration number is big enough that the chain has "forgotten" about $x_{0}$, a Markov chain is not a series of independent draws, and it is relevant to ask whether our MCMC chain has reached a good approximation of independence, or, in other words, how much the variance of the estimator $\widehat{I}_{N}$ suffers from the statistical dependence among the $X_{i}$ s.

\subsubsection{Has the chain forgotten its starting state?}

Although theory on this question is unsatisfying as of today, experimental techniques exist, which help the practitioner assess his chain has converged. The first thing one might try is to launch in parallel 
many chains with different starting points, and check they all give similar results. Besides traceplots, one can plot an online estimate of the mean:

$$
\frac{1}{n} \sum_{i=0}^{n} X_{i}
$$

versus $n=1, \ldots, N$ for all chains and check they all converge towards the same value. If not, then convergence has certainly not been reached. Online estimates of the variance of each chain, of quantiles, etc. can also be useful to plot. There are a number of statistics of the sample that formalize this principle of comparison between many chains. A popular such convergence assessment is known as the Gelman-Rubin diagnosis [18, Section 12.3.4]. See [18, Chapter 12] for a review of other convergence diagnostics.

To cancel the influence of the starting point in the evaluation of $\widehat{I}_{N}$, it is usually advised to discard the first $B$ samples of the chain and replace $\widehat{I}_{N}$ by

$$
\widehat{I}_{N, B}=\frac{1}{N-B+1} \sum_{i=B}^{N} X_{i}
$$

The discarded $B$ samples are called a burn-in sample. Though reducing initialization bias, discarding the first $B$ samples also usually makes the variance of $\widehat{I}_{N, B}$ larger than the variance of $\widehat{I}_{N}$, and so $B$ should be as small as possible to keep the final statistical error low. The choice of an optimal $B$ is an open question. In practice, our personal take is to keep the burn-in below $25 \%$ of the sample, and simply go for a large enough number of samples $N$ that multiple chains with different initializations give similar answers.

\subsubsection{How independent do the samples look?}

After initialization bias, the second convergence issue is that of the independence of the samples. Identifying an MCMC chain converging to $\pi$ to a dynamical system progressively stabilizing at equilibrium, Sokal speaks here of autocorrelation in equilibrium [23]. This is related to the variance of $\widehat{I}_{N}$ in the following way: the more correlation there is between samples $\left(X_{i}\right)$ (the autocorrelation of the chain), the higher the variance of $\widehat{I}_{N}$. The variance of $\widehat{I}_{N}$ will still decrease in $K / N$, but the constant $K$ might be much larger than in the independent case [23].

Again, theoretical answers to this question are not very satisfying as of today, but practical diagnostics exist. Besides plotting the different components of the chain $\left(X_{i}\right)$ versus $i$ and checking independence, the simplest idea is to plot the autocorrelation function of the chain. If $d=1$, it is defined as

$$
\rho(t)=\frac{C(t)}{C(0)}, \text { where } C(t)=\frac{1}{N-t+1} \sum_{i=0}^{N-t}\left(X_{i}-\bar{X}\right)\left(X_{i+t}-\bar{X}\right),
$$

where $\bar{X}=N^{-1} \sum_{i=1}^{N} X_{i}$. If $d>1$ one considers the autocorrelation in each component. The autocorrelation function for $t \geq 0$ should look like a rapidly decreasing exponential starting at 1 and going to 0 , as in Figure 7(b). If not, then one can thin the chain, i.e., keep only one sample every other 10 or higher if necessary. But while this may lead to more independent samples, it also leads to a waste of computational effort and an increase in the variance of the final estimator. If strong autocorrelation is revealed, we recommend to start all over with a different $q$. Indeed, strong autocorrelation often reveals a bad choice in the proposal. Finally, note that if one is only interested in estimating $\int h(x) \pi(x) d x$ for a single $h$, then one should monitor the autocorrelation function of $h(X)$ rather than $X$, which is obtained by replacing each occurrence of $X$ in (10) by $h(X)$. 


\subsubsection{Tuning the proposal distribution of $\mathrm{MH}$}

Consider the Metropolis algorithm of Figure 4. If $q$ proposes only small steps, then the candidates $x$ * will often be accepted since the ratio of the posteriors in Step 4 of Figure 5 will be close to 1 . This will lead to high acceptance but also high autocorrelation: $X_{i+1}$ is almost always in the neighborhood of $X_{i}$, and the chain needs a lot of iterations to cross the space, see Figure 6(a) for a typical traceplot. On the other hand, if $q$ proposes too big steps, then it is likely that they will be rejected, especially if the current state of the chain has a high value of $\pi$. This will lead to the chain being blocked for several iterations, which is an even worse case of autocorrelation, see Figure 6(b) for a typical traceplot. There is thus a compromise to find between small steps and large acceptance rate, and large steps and small acceptance rate. Theory suggests that when the target and the proposals are Gaussian, the acceptance rate to reach minimum variance of $\widehat{I}_{N}$ is approximately 0.5 when $d \leq 2$ and 0.25 otherwise [19, 20]. In the common case where the proposal is Gaussian, practitioners usually proceed as follows: take a proposal with some free stepsize parameter $\sigma$ :

$$
q(y \mid x)=\mathcal{N}\left(y \mid x, \sigma \Sigma_{0}\right)
$$

launch several preliminary runs with different values for $\sigma$, and finally select the value of $\sigma$ that yielded the desired acceptance rate for the final run.

At this stage, we personally advocate the use of a pseudoadaptive strategy: adaptive because it learns a good $\sigma$ along the run, and pseudo because we stop the adaptation before the end of the burnin $^{5}$. While $t \leq B^{\prime}$, where $B^{\prime}$ is smaller than the burn-in length $B$, we advise to adapt $\sigma$ in the following manner: at each iteration $t$, replace $\log (\sigma)$ by

$$
\log (\sigma)+\frac{1}{t^{0.7}}\left(\alpha_{t}-\alpha^{*}\right)
$$

where $\alpha_{t} \in[0,1]$ is the current acceptance rate (the number of accepted samples so far divided by $t$ ), $\alpha^{*}$ is 0.5 if $d \leq 2$ and 0.25 else. The log transformation guarantees that $\sigma$ remains positive. The rationale behind (12) is that if $\alpha_{t}>\alpha^{*}$, then too many steps are accepted, so that the stepsize should be increased, and vice versa. (12) with a large enough $B^{\prime}$ is simply an automatic way to perform the preliminary search for $\sigma$.

\subsubsection{Asymptotic confidence intervals for $\widehat{I}_{N}$}

After having obtained a sample with good properties as discussed previously, we are interested in deriving a confidence interval for $\widehat{I}_{N}-I$. Note that this is not the same as finding a credible interval as in Figure $7(\mathrm{~d})$ : we are here interested in knowing how $\widehat{I}_{N}$ varies when the full sample $\left(X_{1}, \ldots, X_{N}\right)$ varies, being drawn from the same chain. It is a tougher and more open question than with importance sampling. First, we need to check that the chain satisfies a central limit theorem (CLT):

$$
\sqrt{N}\left(\widehat{I}_{N}-I\right) \rightarrow \mathcal{N}\left(0, \sigma_{\text {lim }}^{2}\right)
$$

where the convergence is in distribution. A discussion on the CLT for Markov chains can be found in [18, Section 6.7.2], with a note on MH in [18, Section 7.8.2]. For our needs, we just state that a CLT holds for Metropolis' algorithm with Gaussian proposals and a target with bounded support, see [16, Theorem 4.1]. Now, when a CLT holds, confidence intervals can be built using proper ${ }^{6}$ approximations of $\sigma_{\lim }^{2}$ [10]. These estimators of $\sigma_{\lim }^{2}$ are not difficult to understand, but their description and

\footnotetext{
${ }^{5}$ In Section 4.1, we discuss a fully adaptive algorithm where a similar adaptation is carried out to the end of the run.

${ }^{6} \mathrm{By}$ proper, we mean a sequence $\widehat{\sigma}_{N}$ that converges to $\sigma_{\text {lim }}$ at least in probability.
} 
the conditions for convergence are fairly technical, and we refer the interested reader to [Theorems 1 and 2][10] for recent results on the so-called spectral and overlapping batch means method, our two favourites. A less technical but not up-to-date spectral method is described in [23, Section 3].

\subsection{Implementation tips}

First, MCMC can deal with constrained variables. If the variable is discrete, it is usually easy to find a proposal with the right constrained support. Constrained continuous variables are to be treated differently. One could, for example, include an indicator in the prior that will yield rejection of all points outside the allowed region. But if $\pi$ is large near the boundary of this allowed region, it is likely that the chain will spend time there and thus a lot of points will be rejected only because they do not satisfy the constraint. This is a waste of computational effort, and, if possible, it is usually advised to reparametrize the problem so that it becomes unbounded. If $x$ has to remain positive, then use $\log (x)$. If $x$ has to remain in an interval, then use Argth, for example.

Second, as with most multivariate methods, it is usually better to scale one's variables. The rough idea is that a step in every direction should have the same effect on $\pi$. This should make Gaussians with covariance proportional to the identity reasonable proposals.

Third, whenever manipulating likelihoods, it is advisable to work in the log domain. Computing log-likelihoods is numerically more stable than doing large products of potentially very small numbers, and MCMC code can always be written using only log-likelihoods.

\subsection{A worked out example}

Consider the model of Section 1.1 with a single muon entering the tank: $N_{\mu}=1$. We simulated data with an arrival time $t_{\text {true }}=55$ and an amplitude $A_{\text {true }}=20$. The simulated signal $\mathbf{n}$ is depicted in Figure 7(a).

We place a wide independent Gaussian prior on $t$ and $A$ : $p(t, A)=\mathcal{N}\left(t \mid 100,100^{2}\right) \mathcal{N}\left(A \mid 20,20^{2}\right)$. Let us apply $\mathrm{MH}$ to obtain the posterior $p(t, A \mid \mathbf{n})$. First, let us change variables for $\log (t)$ and $\log (A)$, to avoid dealing with a boundary in $\mathbb{R}^{2} \cdot \log (t)$ and $\log (A)$ roughly have the same scale, so we do not modify them further. Let $T=20000$ iterations, of which we discard the first $B=5000$ as burn-in. We take a Gaussian with covariance $\sigma^{2} I$ as a proposal and apply the pseudoadaptive tuning of (12).

The results ${ }^{7}$ can be seen in Figures 7(a) to 7(e). The autocorrelation function in Figure 7(b) indicates a fairly independent behaviour of the chain. This is confirmed by the traceplot in Figure 7(c) where no clear dependence can be detected by eye.

To obtain marginal distributions of $\pi$, one can simply collect the corresponding coordinate among the $X_{i} \mathrm{~s}$. The marginal histograms here look regular, as shown by the $\log (t)$ marginal in Figure $7(\mathrm{~d})$. The acceptance has approximately reached the optimal $50 \%$ by the end of the burn-in and stabilized there. To check independence from the starting point, we ran 10 chains with uniform initialization on a wide rectangle, and plotted the online sample mean and standard deviations of each chain in Figure 7(f): all chains are consistent.

When reporting a result, the best practice is to give a summary of and make available the entire posterior sample. When $\mathbb{X}$ is high-dimensional, a series of marginal histograms, or $2 \mathrm{D}$ plots of one component versus the other, etc. can be given. In terms of estimation, credible intervals with level $c$ can be computed easily once the sample has been drawn: simply report any interval that contains a proportion $c$ of the samples. Such an interval is computed and given in Figure 7(d), for example.

\footnotetext{
${ }^{7}$ The matlab code used to generate the figures in this section is available on www.stats.ox.ac.uk/ bardenet.
} 


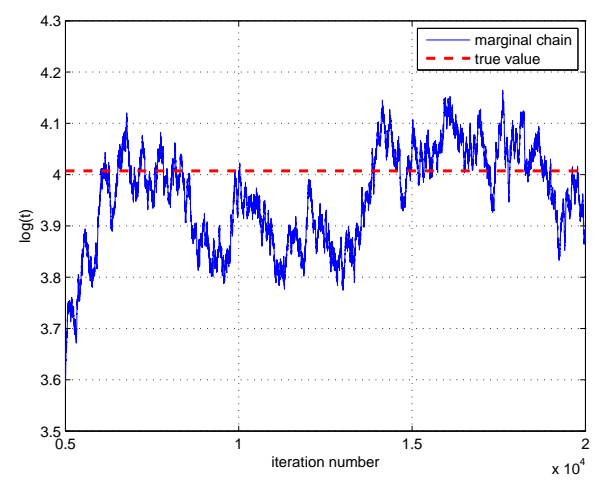

(a) Steps too small, acceptance too high

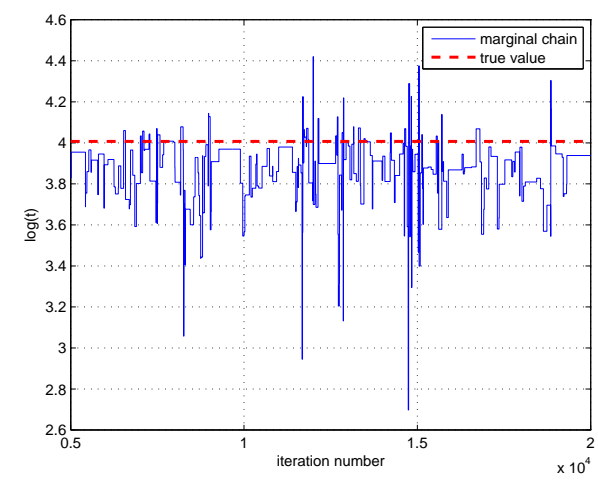

(b) Steps too big, acceptance too low

Figure 6. Two examples high autocorrelation with different causes.

Finally, we give here two highly correlated traceplot examples of badly tuned chains that should ring an alarm, and be compared to the correct behaviour in Figure 7(c). On Figure 6(a), $\sigma$ is too small, leading to an overly slow exploration of the parameter space. On Figure 6(b), $\sigma$ is too large, causing the chain to stay blocked very often.

\subsection{Physics-inspired variants of MH: Gibbs, Langevin and Hamilton}

MH with a simple unimodal proposal (Gaussian, Student) is very generally applicable, but can be enhanced through the use of additional information on the target distribution $\pi$. Although, in our experience, they are rarely used in experimental physics due to the complexity of the models, we quickly review here some popular variants of $\mathrm{MH}$ that were actually inspired by physics.

\subsubsection{The Gibbs sampler}

For $x=\left(x^{1}, \ldots, x^{d}\right) \in \mathbb{X}$ a vector ${ }^{8}$ of length $d$, define $x^{-i}=\left(x^{1}, \ldots, x^{i-1}, x^{i+1}, \ldots x^{d}\right)$. If the posterior is simple enough that it is easy to sample from all conditional distributions $\pi\left(x^{i} \mid x^{-i}\right)$, then one can replace Step 3 of $\mathrm{MH}$ in Figure 5 by a sequence of draws from the conditionals, conditioning on the components already drawn. The acceptance probability $\rho$ in Step 4 in Figure 5 then evaluates to 1 and every proposal is thus accepted. The pseudocode of the Gibbs sampler is given in Figure 8.

The Gibbs sampler is useful in models where the dependencies between variables are non-trivial, but the conditional distributions are easy to sample. Even if this is rarely the case in particle physics, it can happen that some conditional distributions are available, and one might then use Gibbs proposals on some of the variables within an MH scheme. Any concatenation of MCMC steps is permitted, as long as the same concatenation is repeated over and over.

\subsubsection{Langevin diffusion}

If not the conditionals, one might know how to compute the gradient of $\pi$. This is also useful information: although $\mathrm{MH}$ is not an optimization algorithm, visiting all modes of $\pi$ is essential. The Langevin

\footnotetext{
${ }^{8} \mathrm{We}$ use here upper indices to number components of the vector, and keep lower indices for the iteration number in $\mathrm{MH}$
} 


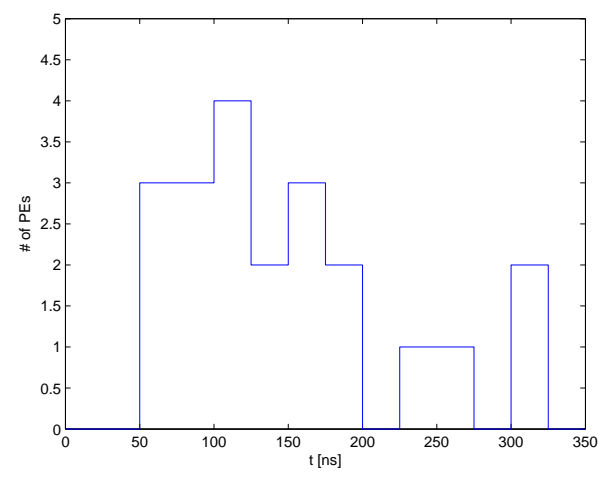

(a) Generated signal

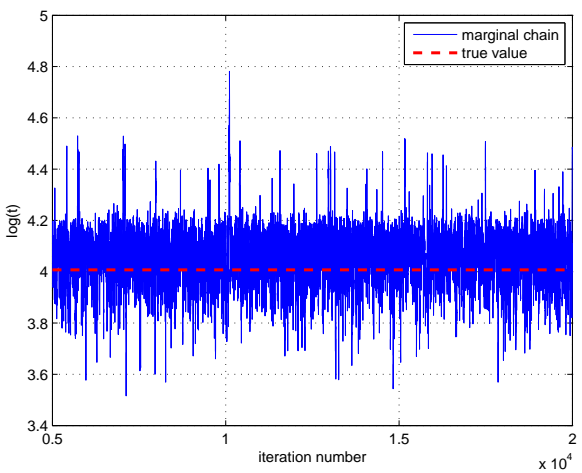

(c) $\log (t)$ chain

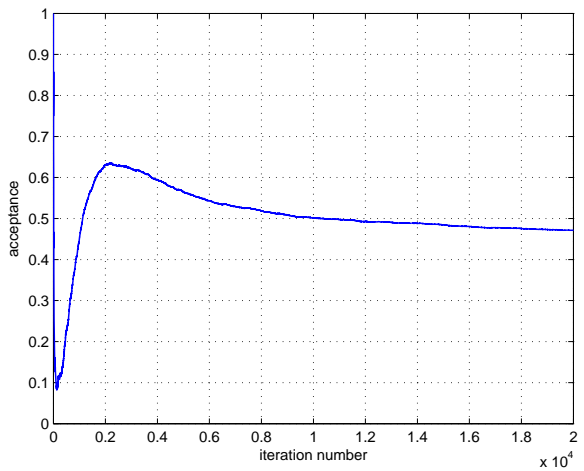

(e) Acceptance

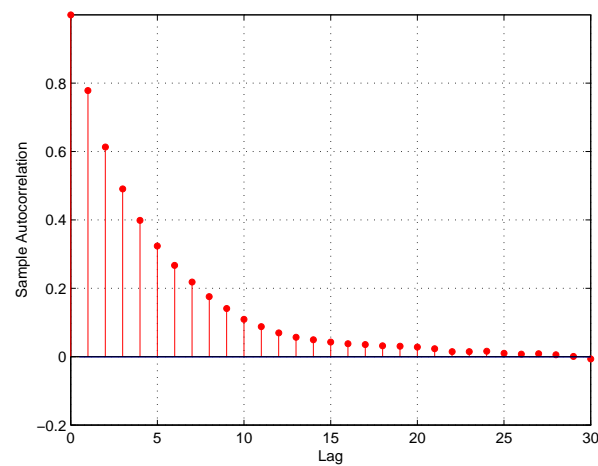

(b) Autocorrelation function

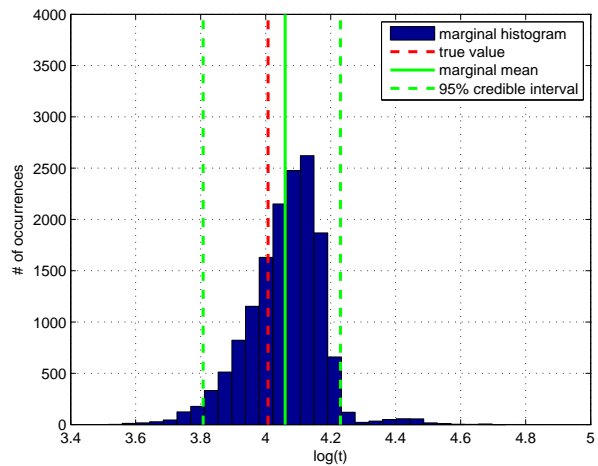

(d) $\log (t)$ histogram

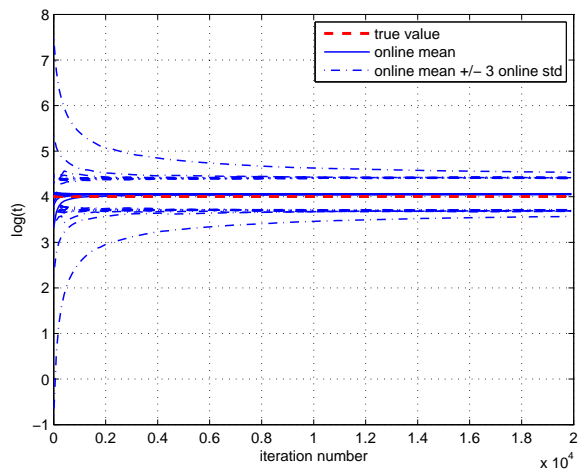

(f) Statistics of 10 independent chains

Figure 7. Results of applying MH in the setup of Section 3.4. See text for details. 


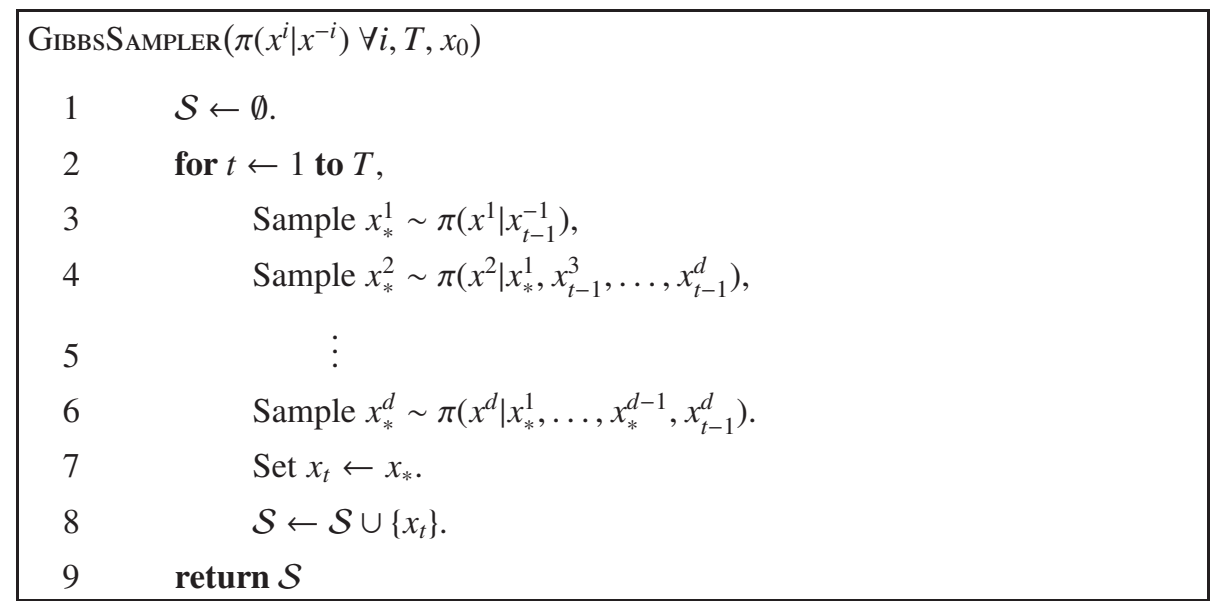

Figure 8. The pseudocode of the Gibbs sampler.

sampler is an $\mathrm{MH}$ sampler with a proposal that is partly deterministic: from $x_{t-1}$, a small step in the direction of the gradient of $\pi$ at $x_{t-1}$ is done, and from there a small Gaussian step is performed. Basically, the Langevin sampler is $\mathrm{MH}$ with proposal

$$
q(y \mid x)=\mathcal{N}\left(y \mid x+\frac{\sigma^{2}}{2} \nabla \log \pi(x), \sigma^{2} I_{d}\right) .
$$

The Langevin sampler is actually inspired by the discretization of a diffusion equation [18, Section 7.8.5 and references therein]. Interestingly, the MH acceptance step can here be interpreted as a correction for the discretization error of the numerical scheme solving the diffusion equation.

Another popular sampler, inspired by mechanics, is Hamiltonian MCMC, or hybrid MCMC [17]. Its main features are that the target is plugged in an energy function, points in $\mathbb{X}$ are interpreted as space coordinates and augmented with an artificial momentum variable, and proposals include a deterministic move along an approximate solution to a system of differential equations, as well as an $\mathrm{MH}$ correction step. Hamiltonian MCMC in its original form also requires a closed form for the gradient, which is again rarely available. Details of the pseudocode of Hamiltonian MCMC are out of the scope of this chapter, but we refer interested readers to [17].

\section{Advanced MCMC methods}

Motivated by the Auger-inspired example of Section 1.1, we review in this section some useful advanced MCMC algorithms. Consider first the case where at least two muons entered the tank: $N_{\mu}>1$. If two muons crossed the tank around the same time, the corresponding amplitude variables $A_{1}$ and $A_{2}$ are likely to be anticorrelated under the posterior: if $A_{1}$ is large and the first muon explains most of the signal, then $A_{2}$ should be small, and vice versa. Thus, having an $\mathrm{MH}$ proposal that detects and uses this correlation, proposing large "diagonal" jumps in the $\left(A_{1}, A_{2}\right)$ subspace, would be more efficient than an independent proposal. In Section 4.1, we present an MCMC algorithm that learns its proposal covariance on the fly. Another difficulty one encounters with the model of Section 1.1 is that in practice $N_{\mu}$ itself is unknown and should be inferred. In Section 4.3 we present an MCMC algorithm that makes proposals across models with different numbers of muons. Finally, the likelihood 
(4) is invariant to the ordering of the muons. This has undesirable effects on marginal inference that MCMC can cope with, as presented in Section 4.2.

\subsection{Adaptive MCMC}

We already mentioned a pseudoadaptive update scheme in (12), where the stepsize of the MH proposal was tuned during a finite number of iterations before being frozen for the rest of the run. Now a legitimate question is whether the asymptotic guarantees on MCMC hold if we let the adaptation run forever?

Adaptive MCMC is a research topic that focuses on designing provably valid MCMC algorithms with proposals that are tuned on the fly until the complete end of the run. The literature is dense, and we single out here one adaptive MCMC algorithm that we use in almost every physics application: the adaptive Metropolis algorithm (AM; [14]).

The pseudocode of AM is given in Figure 9. Note that in practice, it may help to wait for say $10 d$ iterations before using the adapted covariance matrix in the proposal. $\beta$ should be taken in $\left(\frac{1}{2}, 1\right], 1$ meaning freezing the covariance $\Sigma_{t}$ faster. By default, we personally use 0.7 . In the literature, the covariance matrix scale factor, or stepsize, $\sigma$ is often set to $(2.38)^{2} / d$, as it is shown in [19] that this stepsize is optimal in a sense for Gaussian proposals and targets. However, in practice, we recommend the use of an adaptive scheme such as Step 9 in Figure 9, which preserves the convergence of AM. Other adaptive scalings are discussed in [2].

Remark that AM is still called an MCMC algorithm, although the chain is not Markov anymore: given $X_{t-1}, X_{t}$ is still dependent on the previous history of the chain through $\Sigma_{t-1}$. Still, AM was proven to provide an asymptotically unbiased estimator $\widehat{I}_{N}[1]$.

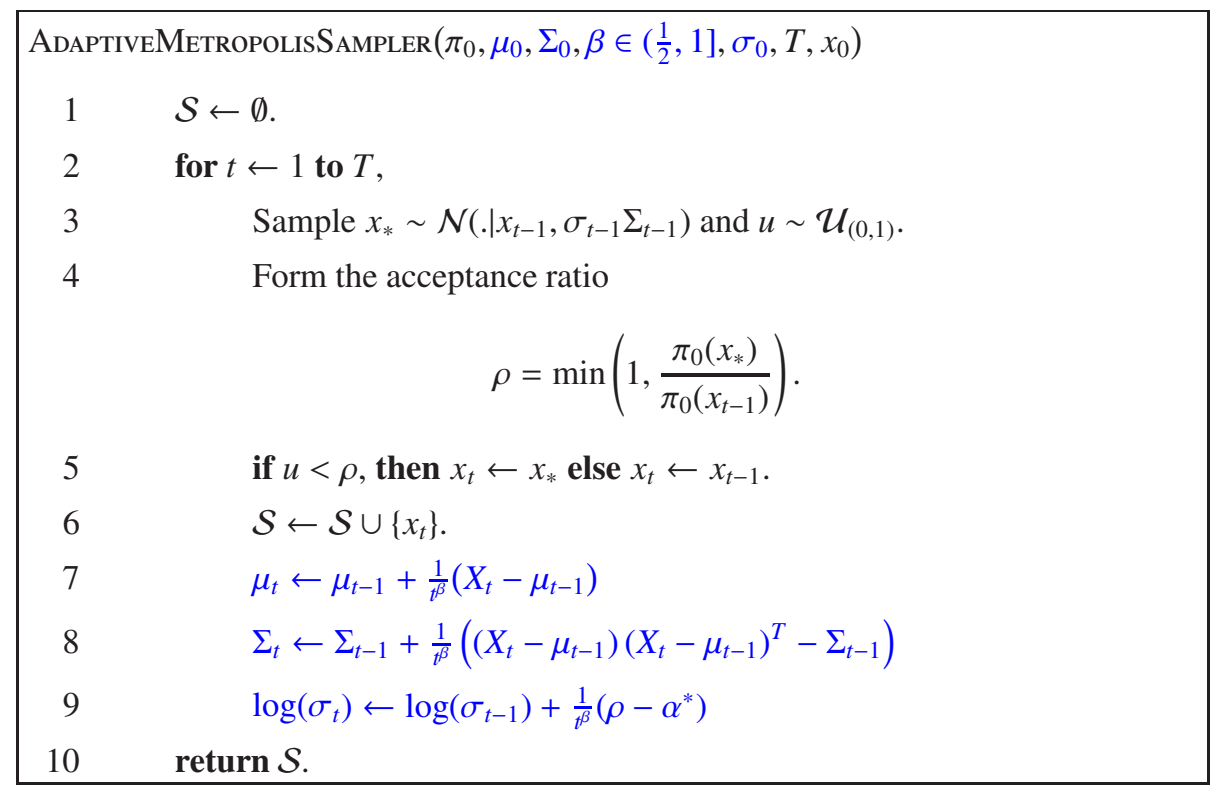

Figure 9. The pseudocode of the adaptive Metropolis algorithm. Modifications with respect to the Metropolis algorithm in Figure 4 are Steps 7 to 9 (in blue). The setting of free parameters $\beta$ and $c$ is discussed in the main text. When $d \leq 2$, we usually set $\alpha^{*}$ to 0.5 , and 0.25 else, but this is only a rule of thumb. 


\subsection{Label switching}

Another feature of the likelihood (4) is that it is invariant to permutations of the muons. In the case where $N_{\mu}=2$, for example,

$$
p\left(\mathbf{n} \mid t_{1}, A_{1}, t_{2}, A_{2}\right)=p\left(\mathbf{n} \mid t_{2}, A_{2}, t_{1}, A_{1}\right) .
$$

If the prior is also invariant, then the posterior $\pi$ inherits the same property. $\pi$ has then as many redundant modes as there are permutations to which it is invariant, and this is undesirable when it comes to marginal inference. Indeed, Figures 10(a) and 10(b) illustrate the challenges when running vanilla AM on the example presented in Figure 7(a). The red variable gets stuck in one of the mixture components, whereas the blue, green, and brown variables visit all the three remaining components, a phenomenon called label switching. As a result, marginal estimates computed for the blue, green, and brown variables are then mostly identical as seen on Figure 10(b). In addition, the shaded ellipses, depicting the marginal posterior covariances of the two parameters $t$ and $A$ of each muon, indicate that the resulting empirical covariance estimate is very broad, resulting in poor efficiency of the adaptive algorithm. Label switching is addressed by so-called relabeling algorithms [5, Chapter 8]. A recent relabeling mechanism that interweaves favorably with AM can be found in [6], along with an application to the Auger model of Section 1.1.

\subsection{Transdimensional problems}

Until now, we have always considered $N_{\mu}$ fixed and known. Now consider the problem of letting $N_{\mu}$ free and trying to estimate it. This problem is termed model selection in statistics, and various Bayesian answers have been given. One full MCMC solution is called reversible jump MCMC (RJM$\mathrm{CMC}$ ) and is due to Green [12]. We will introduce the algorithm through an example here, the generic description of the method and implementation advice can be found in [18, Section 11.2].

Say we know there were $1 \leq N_{\mu} \leq 2$ muons in the tank from some other measurements, and we would like to infer $N_{\mu}$ as well as the corresponding times and amplitudes. Let the prior on $N_{\mu}$ be

$$
p\left(N_{\mu}=1\right)=p\left(N_{\mu}=2\right)=\frac{1}{2} .
$$

We need a chain that targets

$$
\pi(x) \propto p\left(\mathbf{n} \mid N_{\mu}, t_{1}, A_{1}, \ldots, t_{N \mu}, A_{N_{\mu}}\right) p\left(t_{1}, A_{1}, \ldots, t_{N \mu}, A_{N_{\mu}} \mid N_{\mu}\right) p\left(N_{\mu}\right)
$$

and that can take values such as $(1, t, A)$ and $\left(2, t_{1}, A_{1}, t_{2}, A_{2}\right)$. In other words, the state space of the chain would be

$$
\{1\} \times \mathbb{R}^{2} \cup\{2\} \times \mathbb{R}^{4} .
$$

This chain should be able to jump within models, i.e., from $(1, t, A)$ to $\left(1, t^{\prime}, A^{\prime}\right)$ or from $\left(2, t_{1}, A_{1}, t_{2}, A_{2}\right)$ to some other point $\left(2, t_{1}^{\prime}, A_{1}^{\prime}, t_{2}^{\prime}, A_{2}^{\prime}\right)$ with two muons. The chain should also be able to jump across models, i.e., from $(1, t, A)$ to some point $\left(2, t_{1}^{\prime}, A_{1}^{\prime}, t_{2}^{\prime}, A_{2}^{\prime}\right)$ and vice versa. For jumps within the same model, we implement usual MH moves. The main contribution of RJMCMC is a general rule to build jumps across models and the corresponding acceptance probability. The key is to design jumps across models that are likely to be accepted. In our example, to jump from a point $\left(N_{\mu}=1, t, A\right)$ to $\left(N_{\mu}=2, t_{1}, t_{2}, A_{1}, A_{2}\right)$, we may break a muon into two separate muons with roughly half the original amplitude each:

- let $u$ be sampled from a distribution $q(u)$, and let $t_{1}=t-u, t_{2}=t+u$. 


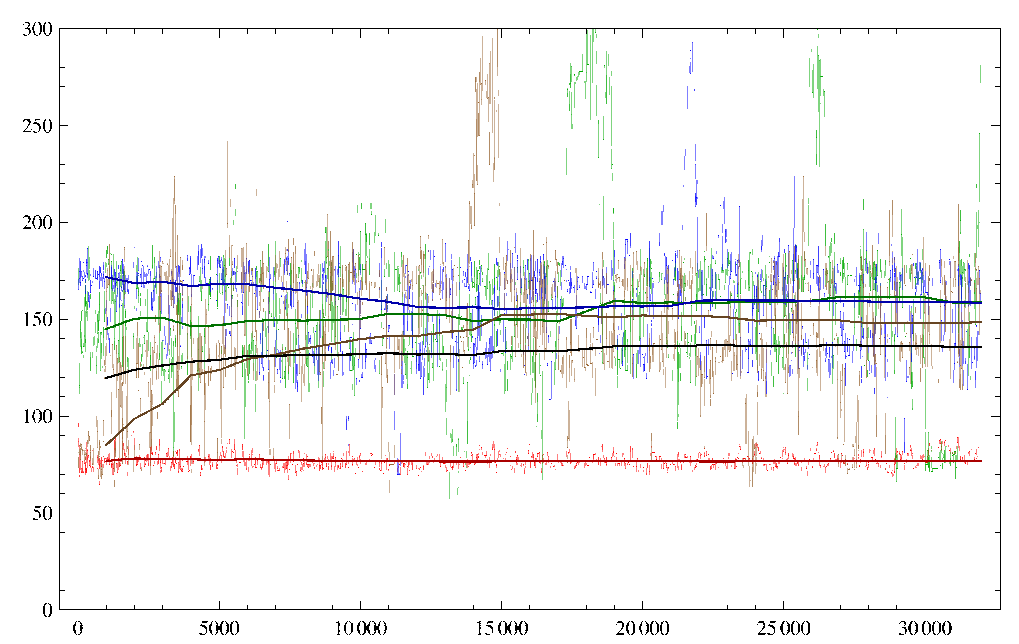

(a) AM: component chains and means

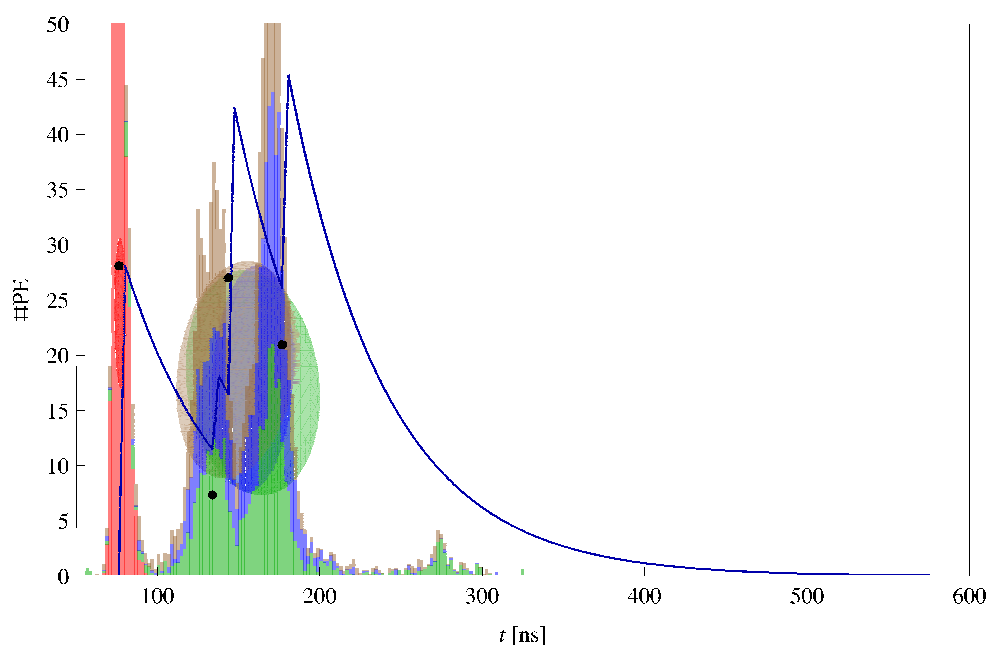

(b) AM: component posteriors

Figure 10. The results of AM on the signal example of Figure 1(b). (a) Three of the four $t$ chains switch position constantly as a result of the target $\pi$ being invariant to permutations of the muons. The corresponding running means (thick lines) converge to similar values. For reference, the thick black line depicts the mean of the coloured thick lines. (b) Label switching causes artificially multimodal marginals. Black dots: the $x$-coordinates are the real time-of-arrival parameters $t$, and the $y$-coordinates are proportional to the amplitudes $A$. Colored ellipses are $\exp (1 / 2)$-level sets of Gaussian distributions: the means are the Bayesian estimates of $(t, A)$ for each muon, and the covariance is the marginal posterior covariance of each $(t, A)$ couple.

- let $v$ be sampled from another distribution $r(v)$, and let the two new amplitudes add up to $A$, by setting $A_{1}=A / 2-v$ and $A_{2}=A / 2+v$. 
This move can be summarized by the application of a one-to-one transformation

$$
T_{1 \rightarrow 2}(t, A, u, v)=\left(t-u, \frac{A}{2}-v, t+u, \frac{A}{2}+v\right),
$$

whose jacobian is $J_{1 \rightarrow 2}=2$. Now that this move from a model with one muon to a model with two muons is fixed, the opposite move is constrained in RJMCMC. A valid choice is the inverse transformation $T^{-1}$, with Jacobian $J_{2 \rightarrow 1}=1 / 2$. Finally, the user has to specify the probabilities $p_{1 \rightarrow 2}$ and $p_{2 \rightarrow 1}$ that a move from $N_{\mu}=1$ to $N_{\mu}=2$ is proposed and vice versa. In the end, the acceptance probability $\rho$ of a move from $(1, t, A)$ to $\left(2, t_{1}, A_{1}, t_{2}, A_{2}\right)$ is given by

$$
\rho=\min \left(1, J_{1 \rightarrow 2} \frac{\pi\left(2, t_{1}, A_{1}, t_{2}, A_{2}\right)}{\pi(1, t, A) q\left(\frac{t_{2}-t_{1}}{2}\right) r\left(\frac{A_{2}-A_{1}}{2}\right)} \frac{p_{2 \rightarrow 1}}{p_{1 \rightarrow 2}}\right),
$$

and the acceptance probability $\rho$ of a move from $\left(2, t_{1}, A_{1}, t_{2}, A_{2}\right)$ to $(1, t, A)$ is given by

$$
\rho=\min \left(1, J_{2 \rightarrow 1} \frac{\pi(1, t, A) q\left(\frac{t_{2}-t_{1}}{2}\right) r\left(\frac{A_{2}-A_{1}}{2}\right)}{\pi\left(2, t_{1}, A_{1}, t_{2}, A_{2}\right)} \frac{p_{1 \rightarrow 2}}{p_{2 \rightarrow 1}}\right) .
$$

RJMCMC is very generic, but transdimensional moves have to be designed with care to be accepted often enough. However, once the chain obtained, inference on $N_{\mu}$ is as easy as on any other parameter: simply count how many times $N_{\mu}=1$ in the chain, divide it by the length of the chain, and you have the posterior probability that $N_{\mu}=1$ ! Reporting the results of an RJMCMC chain can be tricky. Here, one could report the posterior distribution on $N_{\mu}$, and plot the marginals of the other parameters for the most probable values of $N_{\mu}$. Sophisticated summaries have recently been proposed [21], which can compute the probability that one muon in particular is present. Finally, we note that while AM and relabeling can be merged, further including reversible jumps is still research work.

\section{Conclusion and the Monte Carlo ladder}

We reviewed basic Monte Carlo ideas and methods, along with some advanced ones like adaptive MCMC. We tried to give intuition for picking the right method, since none is uniformly powerful, and practical advice on implementations. To sum up the algorithms presented here and point to other important ones that we did not cover, we adapt and complete Murray's integration ladder ${ }^{9}$. Growing item number means higher practical complexity, but also either higher efficiency or wider applicability. Check [18] when no further reference is given.

1. Quadrature,

2. Rejection sampling,

3. Quasi-MC, Importance sampling,

4. MCMC (MH, slice sampling, etc.),

5. Adaptive MCMC [4], hybrid MC [17], tempering methods [11], sequential MC [7], particle MCMC [3].

6. Approximate Bayesian computation (ABC; [9]).

\footnotetext{
${ }^{9} \mathrm{Cf}$. his recommended two-part video lecture at http://videolectures.net/mlss09uk_murray_mcmc/
} 


\section{Appendix: Notations, acronyms, and recommended readings}

Target densities. $\pi$ always denotes the target probability density function, which in Bayesian inference problems is a posterior. Its support is $\mathbb{X} \subset \mathbb{R}^{d}$, and so $d$ denotes the dimension of the problem. $\pi_{0}$ denotes an unnormalized version of $\pi$, formally written as $\pi_{0} \propto \pi$. In Bayesian inference problems, $\pi_{0}$ is often of the form likelihood $\times$ prior.

Estimators. $\widehat{I}_{N}$ always denote the estimator defined in (5), but the $X_{i}$ s used to build it depend on the context. $\widetilde{I}_{N}$ only denotes the importance sampling estimator.

Distributions. We write $X \sim p$ when the probability density function of $X$ is $p$. Used acronyms are summed up for reference in Figure 11.

\begin{tabular}{|r|l|}
\hline MEP & mean posterior (estimate) \\
PE & photoelectron \\
MC & Monte Carlo \\
MCMC & Markov chain Monte Carlo \\
MH & Metropolis-Hastings (algorithm) \\
CLT & central limit theorem \\
AM & adaptive Metropolis (algorithm) \\
\hline
\end{tabular}

Figure 11. Glossary of acronyms, in order of appearance.

For further reading on MC methods, we strongly recommend the textbook [18], to which this tutorial owes a lot. We have tried to refer to specific parts of this book whenever possible. An introduction to MCMC specifically meant for physicists is [23]. While it is a bit outdated now, it still provides insightful and untraditional explanations, especially on assessing MCMC convergence.

\section{Acknowledgments}

I would like to thank the SOS organizers for inviting me to lecture, and for proposing a rich crossdisciplinary programme that generated many interesting discussions. A large part of this tutorial was written while I was working in the Auger group at LAL, Orsay (France).

\section{References}

[1] C. Andrieu, E. Moulines, and P. Priouret. Stability of stochastic approximation under verifiable conditions. SIAM Journal on Control and Optimization, 44:283-312, 2005.

[2] C. Andrieu and J. Thoms. A tutorial on adaptive MCMC. Statistics and Computing, 18:343-373, 2008.

[3] Christophe Andrieu, Arnaud Doucet, and Roman Holenstein. Particle Markov chain Monte Carlo methods. Journal of the Royal Statistical Society B, 2010.

[4] Y. Atchadé, G. Fort, E. Moulines, and P. Priouret. Bayesian Time Series Models, chapter Adaptive Markov chain Monte Carlo: Theory and Methods, pages 33-53. Cambridge Univ. Press, 2011.

[5] R. Bardenet. Towards adaptive learning and inference - Applications to hyperparameter tuning and astroparticle physics. PhD thesis, Université Paris-Sud, 2012.

[6] R. Bardenet and B. Kégl. An adaptive Monte Carlo Markov chain algorithm for inference from mixture signals. In Proceedings of ACAT'11, Journal of Physics: Conference series, 2012.

[7] A. Doucet, N. de Freitas, and N. Gordon. Sequential Monte Carlo in practice. Springer, 2001. 
[8] H. J. Drescher, M. Hladik, S. Ostapchenko, T. Pierog, and Werner K. Parton-based Gribov-Regge theory. Physics Reports, 2001.

[9] P. Fearnhead and D. Prangle. Constructing summary statistics for approximate Bayesian computation: semi-automatic ABC. Journal of the Royal Statistical Society B, 2012.

[10] J. M. Flegal and G. L. Jones. Batch means and spectral variance estimators in Markov chain Monte Carlo. Annals of Statistics, 38(2):1034-1070, 2010.

[11] W.R. Gilks, S. Richardson, and D. Spiegelhalter, editors. Markov Chain Monte Carlo in Practice. Chapman \& Hall, 1996.

[12] P. J. Green. Reversible jump Markov chain Monte Carlo computation and Bayesian model determination. Biometrika, 82(4):711-732, 1995.

[13] G. R. Grimmett and D. R. Stirzaker. Probability and random processes. Oxford science publications, second edition, 1992.

[14] H. Haario, E. Saksman, and J. Tamminen. An adaptive Metropolis algorithm. Bernoulli, 7:223242, 2001.

[15] D. Heck, J. Knapp, J. N. Capdevielle, G. Schatz, and T. Thouw. CORSIKA: A Monte Carlo code to simulate extensive air showers. Technical report, Forschungszentrum Karlsruhe, 1998.

[16] S. F. Jarner and E. Hansen. Geometric ergodicity of Metropolis algorithms. Stochastic processes and their applications, 341-361, 1998.

[17] R. M. Neal. Handbook of Markov Chain Monte Carlo, chapter MCMC using Hamiltonian dynamics. Chapman \& Hall / CRC Press, 2010.

[18] C. P. Robert and G. Casella. Monte Carlo Statistical Methods. Springer-Verlag, New York, 2004.

[19] G. Roberts, A. Gelman, and W. Gilks. Weak convergence of optimal scaling of random walk Metropolis algorithms. The Annals of Applied Probability, 7:110-120, 1997.

[20] G. O. Roberts and J. S. Rosenthal. Optimal scaling for various Metropolis-Hastings algorithms. Statistical Science, 16:351-367, 2001.

[21] A. Roodaki. Signal decompositions using trans-dimensional Bayesian methods. PhD thesis, Supélec, 2012.

[22] D. S. Sivia and J. Skilling. Data Analysis: A Bayesian Tutorial. Oxford University press, second edition, 2006.

[23] A.D. Sokal. Monte Carlo methods in statistical mechanics: Foundations and new algorithms, 1996. Lecture notes at the Cargèse summer school.

[24] D. Wraith, M. Kilbinger, K. Benabed, O. Cappé, J.-F. Cardoso, G. Fort, S. Prunet, and C. P. Robert. Estimation of cosmological parameters using adaptive importance sampling. Phys. Rev. D, 80(2), 2009. 